\title{
Qualitative analysis of an autocatalytic chemical reaction model with decay
}

Jun Zhou

School of Mathematics and Statistics, Southwest University, Chongqing 400715, People's Republic of China

(jzhouwm@gmail.com)

\section{Junping Shi}

Department of Mathematics, College of William and Mary, Williamsburg, VA 23187-8795, USA (jxshix@wm.edu)

(MS received 2 October 2012; accepted 12 February 2013)

\begin{abstract}
In this paper, we revisit a reaction-diffusion autocatalytic chemical reaction model with decay. For higher-order reactions, we prove that the system possesses at least two positive steady-state solutions; hence, it has bistable dynamics similar to the system without decay. For the linear reaction, we determine the necessary and sufficient condition to ensure the existence of a solution. Moreover, in the one-dimensional case, we prove that the positive steady-state solution is unique. Our results demonstrate the drastic difference in dynamics caused by the order of chemical reactions.
\end{abstract}

\section{Introduction}

Autocatalytic chemical reactions are important in many biochemical processes, and various mathematical models of the reactions have been proposed $[1,15,16,24,25$, $30,35]$. In this paper we continue the studies of a reaction-diffusion model of an autocatalytic chemical reaction with decay of the catalyst, which can be written as

$$
A+p B \rightarrow(p+1) B \quad \text { with rate } k_{1} a b^{p}, \quad B \rightarrow C \text { with rate } k_{2} b^{q},
$$

where $p, q \geqslant 1, a$ and $b$ are the concentrations of reactant $A$ and autocatalyst $B$, respectively, and $C$ is an inert product as a kinetic mimic of heat loss. Based on the autocatalytic reaction (1.1), Jakab et al. $[17,18]$ considered the following reactiondiffusion model [18]:

$$
a_{t}=d_{A} \Delta a-k_{1} a b^{p}, \quad b_{t}=d_{B} \Delta b+k_{1} a b^{p}-k_{2} b^{q}, \quad x \in \Omega,
$$

where $d_{A}$ and $d_{B}$ are the diffusion coefficients of $A$ and $B$, respectively, and $\Omega$ is the reactor in $\mathbb{R}^{n}$ for $n \geqslant 1$. The reaction-diffusion system (1.2) is subject to a constant Dirichlet boundary condition

$$
a(x, t)=a_{1}>0, \quad b(x, t)=0, \quad x \in \partial \Omega,
$$

(C) 2014 The Royal Society of Edinburgh 
(representing the reactant $A$ being supplied from outside the reaction zone but the catalyst $B$ not existing outside the boundary $\partial \Omega$ ) and to the initial condition

$$
a(x, 0)=a_{0}(x) \geqslant 0, \quad b(x, 0)=b_{0}(x) \geqslant 0 .
$$

For the case without decay $\left(k_{2}=0\right)$, the existence of positive steady-state solutions and threshold dynamics of (1.2) was studied by Shi and Wang [33] (for $\Omega=\mathbb{R}^{n}$ ) and Jiang and Shi [19] (for bounded $\Omega$ ), while the dynamics of the problem in the whole space $\mathbb{R}^{n}$ with zero boundary condition was considered in $[3,20]$. Moreover, the existence and properties of the travelling wave solution in one-dimensional space were studied by Chen and Qi [6-8], and the stability of the travelling wave solution was recently considered by $\mathrm{Li}$ and $\mathrm{Wu}[21]$.

The system with decay $\left(k_{2}>0\right)$ is considerably harder to analyse due to the asymmetry of the nonlinearity. Recently, Zhao et al. [37] considered the existence, non-existence and bifurcation of positive steady-state solutions as well as basic dynamical properties of (1.2). The aim of this paper is to continue the investigation in [37] to further reveal the structure of the set of positive steady-state solutions of (1.2) with boundary condition (1.3).

With a rescaling $\bar{a}=a / a_{1}, \bar{b}=b / a_{1}, \bar{t}=d_{A} / t$, and dropping the bars of the new variables, the steady-state solutions of (1.2) satisfy

$$
\left.\begin{array}{rl}
\Delta a-\lambda a b^{p}=0, & x \in \Omega, \\
d \Delta b+\lambda a b^{p}-k b^{q}=0, & x \in \Omega, \\
a(x)=1, \quad b(x)=0, & x \in \partial \Omega
\end{array}\right\}
$$

(see [37]), where

$$
d=\frac{d_{B}}{d_{A}}, \quad \lambda=\frac{k_{1} a_{1}^{p}}{d_{A}} \quad \text { and } \quad k=\frac{k_{2} a_{1}^{q-1}}{d_{A}},
$$

$\Omega \subset \mathbb{R}^{n}(n \geqslant 1)$ is a bounded domain with smooth boundary $\partial \Omega$ and the parameters $d, \lambda, k>0$ and $p, q \geqslant 1$.

Because of the non-homogeneous boundary condition of $a$, sometimes it is more convenient to consider the equivalent problem with homogeneous boundary condition. Let $u_{1}=1-a, u_{2}=b$; then $\left(u_{1}, u_{2}\right)$ satisfies

$$
\left.\begin{array}{rlrl}
-\Delta u_{1} & =\lambda\left(1-u_{1}\right) u_{2}^{p}:=f_{1}\left(u_{1}, u_{2}\right), & & x \in \Omega, \\
-d \Delta u_{2} & =\lambda\left(1-u_{1}\right) u_{2}^{p}-k u_{2}^{q}:=f_{2}\left(u_{1}, u_{2}\right), & & x \in \Omega, \\
u_{1}(x) & =u_{2}(x)=0, & & x \in \partial \Omega . .
\end{array}\right\}
$$

Problems (1.5) and (1.6) were studied by Zhao et al. [37] for the case $p, q>1$. They obtained the non-existence and existence of non-trivial steady-state solutions by using energy estimates, the upper-lower solution method and bifurcation theory. Furthermore, they studied the effect of decay order, decay rate and diffusion rates on the dynamical behaviour. Here, we cite one of their results about the existence of positive solutions of problem (1.6) (see [37, theorem 3.4]).

Theorem 1.1. Suppose that $1<p<q$ and $k, \lambda>0$. There then exists $d_{*}>0$ such that, for $0<d<d_{*}$, (1.6) possesses at least one positive solution. 
It has been suggested (see [17-19,33]) that the dynamics of $(1.2)$ is bistable, with two stable non-negative steady-state solutions (one of them is the trivial solution $\left.\left(u_{1}, u_{2}\right)=(0,0)\right)$, and that there exists a threshold set in the phase space separating the basins of attraction of the two stable steady states. Our first result in this paper is to confirm the multiplicity of positive steady-state solutions. More precisely, we prove the following theorem.

TheOREM 1.2. Suppose that $1<p<q, k, \lambda>0$, and suppose that $d_{*}$ is the constant defined in theorem 1.1. Then, for $0<d<d_{*}$, (1.6) possesses at least two distinct positive solutions.

In our second result, we consider (1.6) with $1=p \leqslant q$. In this case we can completely identify the range of parameters for which a positive steady-state solution exists. Moreover, to compare it with the bistable dynamics of $1<p<q$, we show that a unique positive steady-state solution exists for the case $n=1$; thus, the dynamics is monostable. Hence, the order $p$ of the reaction is critical to the asymptotical dynamics. Our second result is stated as the following theorem.

THEOREM 1.3. Suppose that $1=p \leqslant q$, and let $\rho_{1}$ be the first eigenvalue of $-\Delta$ under a homogeneous Dirichlet boundary condition. Then, (1.6) with $q=1$ has a positive solution if and only if $\lambda>k+d \rho_{1}$, and (1.6) with $q>1$ has a positive solution if and only if $\lambda>d \rho_{1}$. Furthermore, the positive solution of (1.6) is unique if $n=1$.

For scalar semilinear equations, the multiplicity for $p>1$ versus the uniqueness for $p=1$ of positive solutions of the logistic-type equation

$$
\left.\begin{array}{rlrl}
-\Delta u & =\lambda(1-u) u^{p}, & & x \in \Omega, \\
u(x) & =0, & & x \in \partial \Omega,
\end{array}\right\}
$$

is well known (see $[26,27,32]$ ), and the results for scalar equations were used for the system (1.6) with $k=0$ in $[19,33]$, as it can be reduced to the scalar case. However, the positive $k$ case of (1.6) cannot be reduced to the scalar case; hence, the proofs of theorems 1.2 and 1.3 are much more difficult. We use Leray-Schauder degree theory to establish the existence of a second positive solution of (1.6) in theorem 1.2, and we use bifurcation theory for the existence/non-existence part in theorem 1.3. The uniqueness of a positive steady-state solution is usually a challenging problem for the reaction-diffusion system, especially for predator-prey-type systems (note that (1.6) is a predator-prey-type system). Here, we adapt an approach in [23] (see also $[4,11,13])$ to prove the uniqueness of a positive steady-state solution.

The paper has the following structure. In $\S 2$, we study the multiplicity of solutions when $1<p<q$ and prove theorem 1.2. In $\S 3$, we study the necessary and sufficient conditions to ensure the existence of a solution when $1=p \leqslant q$ and prove theorem 1.3. For functions $u_{1}, v_{1}, u_{2}, v_{2} \in C(\bar{\Omega})$, we say that $\left(u_{1}, v_{1}\right) \geqslant\left(u_{2}, v_{2}\right)$ if $u_{1}(x) \geqslant u_{2}(x)$ and $v_{1}(x) \geqslant v_{2}(x)$ for any $x \in \Omega$, and that $\left(u_{1}, v_{1}\right)>\left(u_{2}, v_{2}\right)$ if $u_{1}(x)>u_{2}(x)$ and $v_{1}(x)>v_{2}(x)$ for any $x \in \Omega$. 


\section{Multiplicity of positive solutions when $p>1$}

In this section we prove the multiplicity result in theorem 1.2. In the proof, we apply various well-known properties of the Leray-Schauder degree, which can be found in standard references of nonlinear analysis (see, for example, $[5,12,36]$ ). Throughout this section, we always assume that $1<p<q$.

In order to prove theorem 1.2, we need several lemmas. First, we recall the existence of a pair of ordered upper and lower solutions of (1.6), which is shown in [37, proof of theorem 3.4].

Lemma 2.1. Suppose that $1<p<q$ and $k, \lambda>0$. There then exists $d_{*}>0$ such that, for $0<d<d_{*}$, (1.6) has a pair comprising the ordered upper solution $\left(\bar{u}_{1}(x), \bar{u}_{2}(x)\right)$ and the lower solution $\left(\underline{u}_{1}(x), \underline{u}_{2}(x)\right)$, which satisfies

$$
(0,0)<\left(\underline{u}_{1}(x), \underline{u}_{2}(x)\right)<\left(\bar{u}_{1}(x), \bar{u}_{2}(x)\right)<\left(1, d^{-1}\right), \quad x \in \Omega,
$$

and

$$
\begin{aligned}
& -\Delta \bar{u}_{1}>f_{1}\left(\bar{u}_{1}, \bar{u}_{2}\right), \quad x \in \Omega, \\
& -\Delta \underline{u}_{1}<f_{1}\left(\underline{u}_{1}, \underline{u}_{2}\right), \quad x \in \Omega, \\
& -d \Delta \bar{u}_{2}>f_{2}\left(\underline{u}_{1}, \bar{u}_{2}\right), \quad x \in \Omega, \\
& -d \Delta \underline{u}_{2}<f_{2}\left(\bar{u}_{1}, \underline{u}_{2}\right), \quad x \in \Omega, \\
& \left.\bar{u}_{1}=\underline{u}_{1}=\bar{u}_{2}=\underline{u}_{2}=0, \quad x \in \partial \Omega .\right)
\end{aligned}
$$

We use Leray-Schauder degree theory in the proof. Thus, we extend the definition of $f_{1}$ and $f_{2}$ to all real numbers. Define

$$
\tilde{f}_{i}\left(u_{1}, u_{2}\right)=\left\{\begin{array}{ll}
f_{i}\left(u_{1}, u_{2}\right), & u_{2} \geqslant 0, \\
0, & u_{2}<0,
\end{array} \quad i=1,2 .\right.
$$

It is then obvious that $\left(\bar{u}_{1}(x), \bar{u}_{2}(x)\right)$ and $\left(\underline{u}_{1}(x), \underline{u}_{2}(x)\right)$ satisfy $(2.2)$ with $f_{i}=\tilde{f}_{i}$, $i=1,2$. Furthermore, there exists a positive constant $M$ such that, for $i=1,2$,

$$
\left|\tilde{f}_{i}\left(u_{1}^{(1)}, u_{2}^{(1)}\right)-\tilde{f}_{i}\left(u_{1}^{(2)}, u_{2}^{(2)}\right)\right|<M\left(\left|u_{1}^{(1)}-u_{1}^{(2)}\right|+\left|u_{2}^{(1)}-u_{2}^{(2)}\right|\right)
$$

for any $\left(u_{1}^{(1)}, u_{2}^{(1)}\right),\left(u_{1}^{(2)}, u_{2}^{(2)}\right) \in[0,1] \times\left[0, d^{-1}\right]$ and $\left(u_{1}^{(1)}, u_{2}^{(1)}\right) \neq\left(u_{1}^{(2)}, u_{2}^{(2)}\right)$.

Consider the auxiliary problem

$$
\left.\begin{array}{rlrl}
-\Delta u_{1} & =\tilde{f}_{1}\left(u_{1}, u_{2}\right), & & x \in \Omega, \\
-d \Delta u_{2} & =\tilde{f}_{2}\left(u_{1}, u_{2}\right), & & x \in \Omega, \\
u_{1} & =u_{2}=0, & & x \in \partial \Omega .
\end{array}\right\}
$$

Any non-negative solution of (2.4) is then also a solution of (1.6). The next lemma ensures that any solution of (2.4) is non-negative.

Lemma 2.2. Let $u=\left(u_{1}, u_{2}\right)$ be a solution of (2.4); then $\left(u_{1}, u_{2}\right) \geqslant(0,0)$. Thus, any solution of (2.4) is a non-negative solution of (1.6). Also, either $u_{1}=u_{2} \equiv 0$, or $u_{1}(x)>0$ and $u_{2}(x)>0$ for any $x \in \Omega$.

Proof. Set

$$
\Omega^{-}=\left\{x: x \in \Omega, u_{2}(x)<0\right\}
$$


$u_{2}$ then satisfies

$$
-\Delta u_{2}=0, \quad x \in \Omega^{-}, \quad u=0, \quad x \in \partial \Omega^{-} .
$$

So $u_{2} \equiv 0$ for $x \in \Omega^{-}$, i.e. $\Omega^{-}=\emptyset$. So $u_{2} \geqslant 0$ for $x \in \Omega$. From the strong maximum principle of elliptic equations, we have $u_{2} \equiv 0$ or $u_{2}>0$ for $x \in \Omega$.

If $u_{2} \equiv 0$, then $u_{1} \equiv 0$ as well, and the conclusion holds. If $u_{2}>0$ for $x \in \Omega$, we assume that $u_{1}\left(x_{0}\right)=\max _{\bar{\Omega}} u_{1}(x)$. From the first equation of (2.4), we can then see that $u_{1}\left(x_{0}\right) \leqslant 1$ and, consequently, $u_{1}(x) \leqslant 1$ for any $x \in \Omega$. So $u_{1}$ satisfies

$$
-\Delta u_{1} \geqslant 0, \quad x \in \Omega, \quad u_{1}=0, \quad x \in \Omega .
$$

We then get that $u_{1}(x) \geqslant 0$ by the maximum principle of elliptic equations, and that $u_{1}(x)>0$ from the strong maximum principle.

To obtain more detailed information on the iterated sequence generated by the defined upper and lower solutions, we recall the iteration process. For any

$$
u=\left(u_{1}, u_{2}\right) \in C^{1}(\bar{\Omega}) \times C^{1}(\bar{\Omega}),
$$

the problem

$$
\left.\begin{array}{rlrl}
-\Delta v_{1}+M v_{1} & =M u_{1}+\tilde{f}_{1}\left(u_{1}, u_{2}\right), & & x \in \Omega, \\
-d \Delta v_{2}+M v_{2} & =M u_{2}+\tilde{f}_{2}\left(u_{1}, u_{2}\right), & & x \in \Omega, \\
v_{1} & =v_{2}=0, & & x \in \partial \Omega,
\end{array}\right\}
$$

has a unique solution

$$
v=\left(v_{1}, v_{2}\right) \in C^{2+\alpha}(\bar{\Omega}) \times C^{2+\alpha}(\bar{\Omega}), \quad 0<\alpha<1 .
$$

We define $v=\left(v_{1}, v_{2}\right)=T\left(u_{1}, u_{2}\right)$. It is well known that $T$ is a compact operator in $C^{1}(\bar{\Omega}) \times C^{1}(\bar{\Omega})$ and $(2.4)$ is equivalent to the operator equation $\left(u_{1}, u_{2}\right)-$ $T\left(u_{1}, u_{2}\right)=0$.

Consider the following problem:

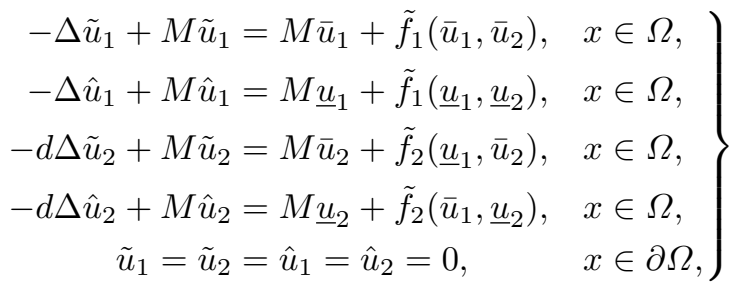

which generates $\left(\tilde{u}_{1}, \tilde{u}_{2}\right)$ and $\left(\hat{u}_{1}, \hat{u}_{2}\right)$ as the first iterated terms from the iteration scheme. By using the maximum principle, we get the following lemma.

LEMMA 2.3. Let $\left(\tilde{u}_{1}, \hat{u}_{1}, \tilde{u}_{2}, \hat{u}_{2}\right)$ be the unique positive solution of (2.6); we then have that

$$
\begin{array}{ccc}
\left(\underline{u}_{1}(x), \underline{u}_{2}(x)\right)<\left(\hat{u}_{1}(x), \hat{u}_{2}(x)\right)<\left(\tilde{u}_{1}(x), \tilde{u}_{2}(x)\right)<\left(\bar{u}_{1}(x), \bar{u}_{2}(x)\right), & x \in \Omega, \\
\left(\frac{\partial \tilde{u}_{1}}{\partial \nu}(x), \frac{\partial \tilde{u}_{2}}{\partial \nu}(x)\right)<\left(\frac{\partial \hat{u}_{1}}{\partial \nu}(x), \frac{\partial \hat{u}_{2}}{\partial \nu}(x)\right), & & x \in \partial \Omega,
\end{array}
$$

where $\nu$ is the unit outward normal on $\partial \Omega$. 
Proof. Since $\hat{u}_{1}$ satisfies

$$
-\Delta \hat{u}_{1}+M \hat{u}_{1}=M \underline{u}_{1}+\tilde{f}_{1}\left(\underline{u}_{1}, \underline{u}_{2}\right)>M \underline{u}_{1}-\Delta \underline{u}_{1}, \quad x \in \Omega,
$$

we obtain that

$$
-\Delta w+M w>0, \quad x \in \Omega, \quad w=0, \quad x \in \partial \Omega,
$$

where $w=\hat{u}_{1}(x)-\underline{u}_{1}(x)$. So, $w>0$ in $\Omega$ by the strong maximum principle of elliptic equations, i.e. $\hat{u}_{1}(x)>\underline{u}_{1}(x), x \in \Omega$. Similarly, we can prove that $\hat{u}_{2}(x)>\underline{u}_{2}(x)$, $x \in \Omega$. In the same way as above, we can prove that $\left(\tilde{u}_{1}(x), \tilde{u}_{2}(x)\right)<\left(\bar{u}_{1}(x), \bar{u}_{2}(x)\right)$, $x \in \Omega$.

Next, we set $z_{1}(x)=\tilde{u}_{1}(x)-\hat{u}_{1}(x), z_{2}(x)=\tilde{u}_{2}(x)-\hat{u}_{2}(x)$. Since

$$
\begin{aligned}
\tilde{f}_{1}\left(\bar{u}_{1}, \bar{u}_{2}\right)-\tilde{f}_{1}\left(\underline{u}_{1}, \underline{u}_{2}\right) & =\left(\tilde{f}_{1}\left(\bar{u}_{1}, \bar{u}_{2}\right)-\tilde{f}_{1}\left(\bar{u}_{1}, \underline{u}_{2}\right)\right)+\left(\tilde{f}_{1}\left(\bar{u}_{1}, \underline{u}_{2}\right)-\tilde{f}_{1}\left(\underline{u}_{1}, \underline{u}_{2}\right)\right) \\
& >-M\left(\bar{u}_{1}-\underline{u}_{1}\right), \\
\tilde{f}_{2}\left(\underline{u}_{1}, \bar{u}_{2}\right)-\tilde{f}_{2}\left(\bar{u}_{1}, \underline{u}_{2}\right) & =\left(\tilde{f}_{2}\left(\underline{u}_{1}, \bar{u}_{2}\right)-\tilde{f}_{2}\left(\underline{u}_{1}, \underline{u}_{2}\right)\right)+\left(\tilde{f}_{2}\left(\underline{u}_{1}, \underline{u}_{2}\right)-\tilde{f}_{2}\left(\bar{u}_{1}, \underline{u}_{2}\right)\right) \\
& >-M\left(\bar{u}_{2}-\underline{u}_{2}\right),
\end{aligned}
$$

$\left(z_{1}, z_{2}\right)$ satisfies

$$
\begin{aligned}
& -\Delta z_{1}+M z_{1}>0, \quad x \in \Omega, \quad z_{1}=0, \quad x \in \partial \Omega, \\
& -\Delta z_{2}+M z_{2}>0, \quad x \in \Omega, \quad z_{2}=0, \quad x \in \partial \Omega .
\end{aligned}
$$

By the strong maximum principle and the Hopf lemma, we then obtain that

$$
z_{i}(x)>0, \quad x \in \Omega, \quad \frac{\partial z_{i}}{\partial \nu}<0, \quad x \in \partial \Omega, \quad i=1,2 .
$$

The properties proved in lemma 2.3 allow us to define a convex subset in the function space, which will be useful in the proof. Let

$$
E=\left\{u: u=\left(u_{1}, u_{2}\right) \in C^{1}(\bar{\Omega}) \times C^{1}(\bar{\Omega}), u_{i}(x)=0, x \in \partial \Omega, i=1,2\right\} .
$$

Then, $E$ is a Banach space with norm $\left\|\left(u_{1}, u_{2}\right)\right\|_{E}=\left\|u_{1}\right\|_{C^{1}}+\left\|u_{2}\right\|_{C^{1}}$, and, for any $u=\left(u_{1}, u_{2}\right) \in E$, if $\underline{u}_{i}(x) \leqslant u_{i}(x) \leqslant \bar{u}_{i}(x), x \in \Omega, i=1,2$, then there exists a positive constant $M_{1}$ such that $\|T u\|_{E}<M_{1}$. Moreover, we define

$$
\begin{aligned}
U=\left\{u=\left(u_{1}, u_{2}\right)\right. & \in E: \hat{u}_{i}(x)<u_{i}(x)<\tilde{u}_{i}(x), x \in \Omega \\
& \left.\frac{\partial \tilde{u}_{i}}{\partial \nu}(x)<\frac{\partial u_{i}}{\partial \nu}(x)<\frac{\partial \hat{u}_{i}(x)}{\partial \nu}, x \in \partial \Omega,\|u\|_{E}<M_{1}, i=1,2\right\} ;
\end{aligned}
$$

then $U$ is an open convex set in $E$. Furthermore, we have the following lemma.

Lemma 2.4. The set $U$ defined in (2.7) satisfies $T(\bar{U}) \subset U$ and

$$
\operatorname{deg}(I-T, U,(0,0))=1 .
$$


Proof. Similarly to the proof of lemma 2.3, we can get the first conclusion. The second conclusion follows from well-known properties of Leray-Schauder degree theory (see, for example, [36, theorem 6.3.1] or [12]).

To find fixed points for $(2.5)$, we consider the following homotopy problem:

$$
\left.\begin{array}{rlrl}
-\Delta u_{1}+t M u_{1} & =t\left[M u_{1}+\tilde{f}_{1}\left(u_{1}, u_{2}\right)\right], & & x \in \Omega, \\
-d \Delta u_{2}+t M u_{2} & =t\left[M u_{2}+\tilde{f}_{2}\left(u_{1}, u_{2}\right)\right], & & x \in \Omega, \\
u_{1} & =u_{2}=0, & & x \in \partial \Omega,
\end{array}\right\}
$$

where $t \in[0,1]$. Problem (2.8) is then equivalent to the operator equation $\left(u_{1}, u_{2}\right)-$ $T_{t}\left(u_{1}, u_{2}\right)=(0,0)$, where $\left(v_{1}, v_{2}\right)=T_{t}\left(u_{1}, u_{2}\right)$ is defined by the unique solution of

$$
\left.\begin{array}{rlrl}
-\Delta v_{1}+t M v_{1} & =t\left[M u_{1}+\tilde{f}_{1}\left(u_{1}, u_{2}\right)\right], & & x \in \Omega, \\
-d \Delta v_{2}+t M v_{2} & =t\left[M u_{2}+\tilde{f}_{2}\left(u_{1}, u_{2}\right)\right], & & x \in \Omega, \\
u_{1} & =u_{2}=0, & & x \in \partial \Omega .
\end{array}\right\}
$$

It is well known that $T_{t}:[0,1] \times E \rightarrow E$ is a compact operator, and, furthermore, we have the following lemma.

LEMMA 2.5. Let $u=\left(u_{1}, u_{2}\right)$ be a non-zero solution of (2.8); the following then hold:

(i) there exists a constant $R>0$ such that $\|u\|_{E}<R$;

(ii) there exists a constant $\delta>0$ such that $\|u\|_{E}>\delta$.

Proof. In the proof, we use $C$ as a positive constant, which may change value from line to line.

(i) Similarly to [37, proof of lemma 2.1], we obtain that there exists a positive constant $R_{0}$ such that

$$
\max _{\bar{\Omega}}\left|u_{i}\right|<R_{0}, \quad i=1,2 .
$$

By Sobolev's embedding theorem and $L^{r}$-estimates, we have that

$$
\begin{aligned}
\left\|u_{i}\right\|_{C^{1+\alpha}} & \leqslant C\left(\left\|u_{1}\right\|_{W^{2, r}}+\left\|u_{2}\right\|_{W^{2, r}}\right) \\
& \leqslant C\left(\left\|\tilde{f}_{1}\left(u_{1}, u_{2}\right)\right\|_{L^{r}}+\left\|\tilde{f}_{2}\left(u_{1}, u_{2}\right)\right\|_{L^{r}}\right),
\end{aligned}
$$

where $r$ is a positive constant such that $2-n / r>1+\alpha$. Therefore, there exists a constant $R>0$ satisfying that $\|u\|_{E}<R$.

(ii) If $R>0$ does not satisfy $\|u\|_{E}<R$, then there exists a sequence $\left\{t_{m}\right\}_{m=1}^{+\infty} \subset$ $[0,1]$ such that the solution $u_{m}=\left(u_{1 m}, u_{2 m}\right)$ of $(2.8)$ with $t=t_{m}$ satisfies $\left\|u_{m}\right\|_{E}>$ $0,\left\|u_{m}\right\|_{E} \rightarrow 0$ as $m \rightarrow \infty$. On the other hand, we have that

$$
\begin{aligned}
\left\|u_{m}\right\|_{E} & \leqslant C\left(\left\|\tilde{f}_{1}\left(u_{1 m}, u_{2 m}\right)\right\|_{L^{r}}+\left\|\tilde{f}_{2}\left(u_{1 m}, u_{2 m}\right)\right\|_{L^{r}}\right) \\
& \leqslant C\left(\max _{\bar{\Omega}}\left|\tilde{f}_{1}\left(u_{1 m}, u_{2 m}\right)\right|+\max _{\bar{\Omega}}\left|\tilde{f}_{2}\left(u_{1 m}, u_{2 m}\right)\right|\right) \\
& \leqslant C\left\|u_{2 m}\right\|_{\infty}^{p} \leqslant C\left\|u_{m}\right\|_{E}^{p},
\end{aligned}
$$

which is a contradiction, with $\left\|u_{m}\right\|_{E} \rightarrow 0$ as $m \rightarrow \infty$ and $p>1$. 


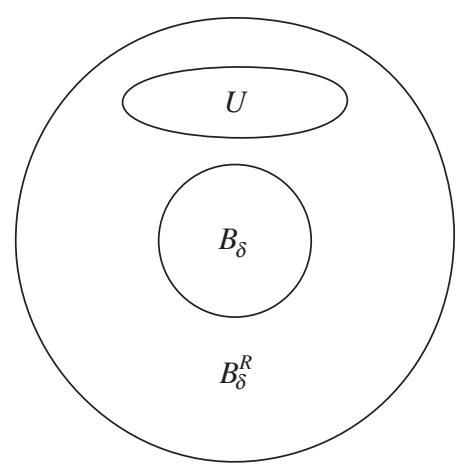

Figure 1. Illustration of the sets in the proof of theorem 1.2.

We are now ready to prove theorem 1.2.

Proof of theorem 1.2. By lemma 2.4 and Schauder's fixed-point theorem, (2.4) has a positive solution in $U$ and

$$
\operatorname{deg}(I-T, U,(0,0))=1 .
$$

By lemma 2.5(i), we know that there exists a ball $B_{R}=B_{R}(0,0) \supset \bar{U}$, and $(0,0) \notin$ $\left(I-T_{t}\right)\left(\partial B_{R}\right)$ for all $t \in[0,1]$. By lemma 2.5(ii), we know that there exists a ball $B_{\delta}=B_{\delta}(0,0)$ such that $\bar{B}_{\delta} \subset B_{R}, \bar{B}_{\delta} \cap \bar{U}=\emptyset$ and $(0,0) \notin\left(I-T_{t}\right)\left(\partial B_{\delta}\right)$ for all $t \in[0,1]$.

Let $B_{\delta}^{R}=B_{R} \backslash \bar{B}_{\delta}$; then (see figure 1)

$$
B_{\delta}^{R}=U \cup \partial U \cup\left(B_{\delta}^{R} \backslash \bar{U}\right) .
$$

By the additivity of the Leray-Schauder degree and the fact, from lemma 2.4, that $(0,0) \notin(I-T)(\partial U)$, we get that

$$
\begin{aligned}
\operatorname{deg}\left(I-T, B_{\delta}^{R},(0,0)\right) & =\operatorname{deg}(I-T, U,(0,0))+\operatorname{deg}\left(I-T, B_{\delta}^{R} \backslash \bar{U},(0,0)\right) \\
& =1+\operatorname{deg}\left(I-T, B_{\delta}^{R} \backslash \bar{U},(0,0)\right) .
\end{aligned}
$$

By the homotopy invariance of the Leray-Schauder degree and the fact that there is no solution of $\left(I-T_{0}\right)\left(u_{1}, u_{2}\right)=(0,0)$ in $B_{\delta}^{R}$, we have that

$$
\operatorname{deg}\left(I-T, B_{\delta}^{R},(0,0)\right)=\operatorname{deg}\left(I-T_{0}, B_{\delta}^{R},(0,0)\right)=0 .
$$

From (2.10) and (2.11), we obtain that

$$
\operatorname{deg}\left(I-T, B_{\delta}^{R} \backslash \bar{U},(0,0)\right)=-1 .
$$

Therefore, (2.4) has another non-zero solution in $B_{\delta}^{R} \backslash \bar{U}$, which we denote by $\breve{u}=\left(\breve{u}_{1}, \breve{u}_{2}\right)$. From the construction of $B_{\delta}^{R}$, we know that $\|\breve{u}\|_{E}>\delta$ and, from the definition of $\tilde{f}_{1}$ and $\tilde{f}_{2},\left(\breve{u}_{1}, \breve{u}_{2}\right)>0$ for $i=1,2$ by lemma 2.2. Hence, (1.6) has one positive solution in $U$ and another positive solution in $B_{\delta}^{R} \backslash \bar{U}$, and the theorem is proved. 


\section{Existence and bifurcation of positive solutions when $p=1$}

In this section we prove the existence part of theorem 1.3, and we prove the uniqueness part of theorem 1.3 when $n=1$ in $\S 4$. Here, we consider (1.6) with $q \geqslant p=1$, i.e. the following system of semilinear elliptic equations:

$$
\left.\begin{array}{rlrl}
-\Delta u_{1} & =\lambda u_{2}-\lambda u_{1} u_{2}, & & x \in \Omega, \\
-d \Delta u_{2} & =\lambda u_{2}-\lambda u_{1} u_{2}-k u_{2}^{q}, & & x \in \Omega, \\
u_{1} & =u_{2}=0, & & x \in \partial \Omega .
\end{array}\right\}
$$

For later discussion, we establish some notation (see, for example, [2]), which will be used throughout this section. For any $q(x)$ in $C(\bar{\Omega})$ and $d>0$, the linear eigenvalue problem

$$
\left.\begin{array}{rlrl}
-d \Delta u+q(x) u & =\rho u, & & x \in \Omega \\
u & =0, & & x \in \partial \Omega
\end{array}\right\}
$$

has an infinite sequence of eigenvalues, $\rho_{1}<\rho_{2} \leqslant \rho_{3} \leqslant \cdots$, which are bounded from below. It is also known that the principal eigenvalue

$$
\rho_{1}=\rho_{1}(-d \Delta+q(x))
$$

is simple and any solution of (3.2) with $\rho=\rho_{1}(-d \Delta+q(x))$ is a constant multiple of an eigenfunction that does not change sign in $\Omega$ and whose normal derivatives never vanish on the boundary $\partial \Omega$. Furthermore, $\rho_{1}$ is strictly increasing in the sense that $q_{1}(x), q_{2}(x) \in C(\bar{\Omega}), q_{1}(x) \leqslant q_{2}(x) ; q_{1}(x) \not \equiv q_{2}(x)$ implies that $\rho_{1}\left(-d \Delta+q_{1}(x)\right)<$ $\rho_{1}\left(-d \Delta+q_{2}(x)\right)$. Furthermore, according to the variational characterization for $\rho_{1}(-d \Delta+q(x))$, we know that

$$
\rho_{1}(-d \Delta+q(x))=\inf _{\varphi \in H_{0}^{1}(\Omega), \varphi \neq 0} \frac{\int_{\Omega}\left(d|\nabla \varphi|^{2}+q \varphi^{2}\right) \mathrm{d} x}{\int_{\Omega} \varphi^{2} \mathrm{~d} x} .
$$

In particular, $\rho_{1}=\rho_{1}(-\Delta)$ is the principal eigenvalue of $-\Delta$ subject to the homogeneous Dirichlet boundary condition, and the corresponding positive eigenfunction is denoted by $\omega(x)$. We normalize the eigenfunction such that $\omega(x)$ satisfies

$$
-\Delta \omega=\rho_{1} \omega, \quad x \in \Omega, \quad \omega=0, \quad x \in \partial \Omega, \quad \int_{\Omega} \omega^{2} \mathrm{~d} x=1 .
$$

From the arguments in lemma 2.2, a non-negative solution of $(3.1)$ is either $(0,0)$ or a positive solution. A positive solution of (3.1) satisfies the following a priori estimates.

Lemma 3.1. Suppose that $\left(u_{1}, u_{2}\right)$ is a positive solution of (3.1). Then

$$
0<d u_{2}(x)<u_{1}(x)<1, \quad 0<u_{2}(x)<\frac{1}{d}, \quad x \in \Omega .
$$

Proof. Let $x_{0} \in \Omega$ such that $u_{1}\left(x_{0}\right)=\max _{\bar{\Omega}} u_{1}(x)$. Then $-\Delta u_{1}\left(x_{0}\right) \geqslant 0$, and, from the first equation of (3.1), it becomes apparent that $0 \leqslant \lambda\left(1-u_{1}\left(x_{0}\right)\right) u_{2}\left(x_{0}\right)$. Consequently, we have that $u_{1}\left(x_{0}\right) \leqslant 1$. That $u_{1}(x)<1$ in $\Omega$ follows from the strong maximum principle. 
By a direct calculation, we find from (3.1) that

$$
-\Delta\left(d u_{2}-u_{1}\right)=-k u_{2}^{q}<0, \quad x \in \Omega, \quad d u_{2}-u_{1}=0, \quad x \in \partial \Omega .
$$

So, $d u_{2}(x)<u_{1}(x), x \in \Omega$ by the strong maximum principle, which implies (3.6).

In the following discussion, we distinguish between the two cases, $q=1$ and $q>1$. First we consider (3.1) with $q=1$, i.e. the system

$$
\left.\begin{array}{rlrl}
-\Delta u_{1} & =\lambda u_{2}-\lambda u_{1} u_{2}, & & x \in \Omega, \\
-d \Delta u_{2} & =\lambda u_{2}-\lambda u_{1} u_{2}-k u_{2}, & & x \in \Omega, \\
u_{1} & =u_{2}=0, & & x \in \partial \Omega .
\end{array}\right\}
$$

In the following we consider positive solutions of (3.8) in the space $X^{2}$, where $X=W^{2, r}(\Omega) \cap W_{0}^{1, r}(\Omega)$ for $r>n$. From the smoothness of the nonlinearities in $(3.8)$, such solutions indeed belong to $\left[C^{2+\alpha}(\bar{\Omega})\right]^{2}$.

Our existence/non-existence and bifurcation result for (3.8) is as follows.

Theorem 3.2. Suppose that $d, k>0$. Then (3.8) has no positive solution if $\lambda \leqslant$ $k+d \rho_{1}$, and (3.8) has at least one positive solution if $\lambda>k+d \rho_{1}$. Moreover, the following hold.

(1) $\lambda=k+d \rho_{1}$ is a bifurcation value of (3.8), where positive solutions bifurcate from the line of trivial solutions $\Gamma_{0}=\{(\lambda, 0,0): \lambda>0\}$, and $\lambda=k+d \rho_{1}$ is the unique bifurcation value for which positive solutions bifurcate from $\Gamma_{0}$.

(2) Near $\left(\lambda, u_{1}, u_{2}\right)=\left(k+d \rho_{1}, 0,0\right)$, all positive solutions of (3.8) lie on a smooth curve $\Gamma_{1}=\left\{\left(\lambda(s), u_{1}(s, x), u_{2}(s, x)\right): s \in(0, \delta), x \in \Omega\right\}$ for some $\delta>0$, with

$$
\begin{aligned}
\lambda(s) & =k+d \rho_{1}+s d\left(k+d \rho_{1}\right)^{2} \int_{\Omega} \omega^{3} \mathrm{~d} x+s \theta(s), \\
u_{1}(s) & =s\left(k+d \rho_{1}\right) d \omega+s \phi_{1}(s), \\
u_{2}(s) & =s \omega+s \phi_{2}(s),
\end{aligned}
$$

where $\left(\left(k+d \rho_{1}\right) d \omega, \omega\right)$ is a positive solution of the following eigenvalue problem with $\rho=0$ :

$$
\left.\begin{array}{rlrl}
\Delta \xi+\lambda \eta & =\rho \xi, & & x \in \Omega, \\
d \Delta \eta+\lambda \eta-k \eta & =\rho \eta, & & x \in \Omega, \\
\xi=\eta & =0, & & x \in \partial \Omega,
\end{array}\right\}
$$

$\theta$ is a smooth function defined on $(0, \delta)$ such that $\theta(0)=\theta^{\prime}(0)=0$, and $\phi_{1}, \phi_{2}$ are smooth functions from $(0, \delta)$ into a subspace of $X^{2}$ that complements $\operatorname{span}\left\{\left(\left(k+d \rho_{1}\right) d \omega, \omega\right)\right\}$ such that $\phi_{i}(0)=\phi_{i}^{\prime}(0)=0$ for $i=1,2$.

(3) $\Gamma_{1}$ is contained in a connected component $\Sigma_{1}$ of the set of positive solutions of (3.8) in $\mathbb{R}^{+} \times X^{2}$ such that

$$
P_{\lambda} \Sigma_{1}=\left(k+d \rho_{1}, \infty\right), \quad P_{u_{1}} \Sigma_{1} \subset(0,1), \quad P_{u_{2}} \Sigma_{1} \subset(0,1 / d),
$$



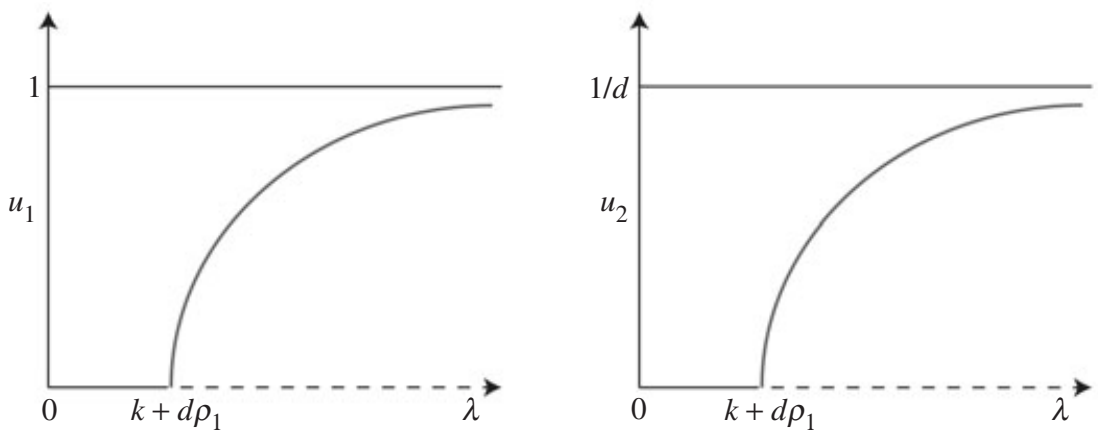

Figure 2. Possible global bifurcation diagram of positive solutions of (3.8).

where $P_{\lambda}$ stands for the projection operator into the $\lambda$-component of $\mathbb{R}^{+} \times X^{2}$, and $P_{u_{i}}$ stands for the projection operator into the $u_{i}$-component, $i=1,2$ (see figure 2).

Proof. We divide the proof into several parts.

(1) (Non-existence.) We prove that $\lambda>k+d \rho_{1}$ is a necessary condition to ensure that (3.8) has a positive solution. In fact, multiplying the second equation of (3.8) by $\omega(x)$, and then integrating the result by parts over $\Omega$, we can obtain the following equality by using $-\Delta \omega=\rho_{1} \omega$ :

$$
\lambda \int_{\Omega} u_{1} u_{2} w \mathrm{~d} x=\left(\lambda-k-d \rho_{1}\right) \int_{\Omega} u_{2} \omega \mathrm{d} x .
$$

So $\lambda>k+d \rho_{1}$ if $\left(\lambda, u_{1}, u_{2}\right)$ is a positive solution of (3.8).

(2) (Local bifurcation.) By linearizing (3.8) at $(\lambda, 0,0)$, we obtain the eigenvalue problem (3.9). A necessary condition for bifurcation is that the principal eigenvalue of (3.9) is 0 , which occurs if $\lambda=k+d \rho_{1}$, and the corresponding eigenfunction is $(\psi(x), \omega(x))$, where $\omega(x)$ is defined in $(3.5)$ and $\psi(x)=\left(k+d \rho_{1}\right)(-\Delta)^{-1}(\omega(x))=$ $\left(k+d \rho_{1}\right) d \omega(x)$.

We apply a bifurcation result of Crandall and Rabinowitz [9] at $(\lambda, 0,0)$. For fixed $d, k>0$, define a nonlinear mapping $F: \mathbb{R} \times X^{2} \rightarrow Y^{2}$, where $Y=L^{r}(\Omega)$, by

$$
F\left(\lambda, u_{1}, u_{2}\right)=\left(\begin{array}{c}
\Delta u_{1}+\lambda u_{2}-\lambda u_{1} u_{2} \\
d \Delta u_{2}+\lambda u_{2}-\lambda u_{1} u_{2}-k u_{2}
\end{array}\right) .
$$

We consider the bifurcation at $\left(\lambda, u_{1}, u_{2}\right)=\left(k+d \rho_{1}, 0,0\right)$. From straightforward calculations, we find the Fréchet derivatives of $F$ to be

$$
\begin{gathered}
F_{\left(u_{1}, u_{2}\right)}\left(\lambda, u_{1}, u_{2}\right)[\xi, \eta]=\left(\begin{array}{c}
\Delta \xi-\lambda u_{2} \xi+\lambda \eta-\lambda u_{1} \eta \\
d \Delta \eta-\lambda u_{2} \xi+\lambda \eta-\lambda u_{1} \eta-k \eta
\end{array}\right), \\
F_{\lambda}\left(\lambda, u_{1}, u_{2}\right)=\left(\begin{array}{c}
u_{2}-u_{1} u_{2} \\
u_{2}-u_{1} u_{2}
\end{array}\right), \quad F_{\lambda\left(u_{1}, u_{2}\right)}\left(\lambda, u_{1}, u_{2}\right)[\xi, \eta]=\left(\begin{array}{l}
-u_{2} \xi+\eta-u_{1} \eta \\
-u_{2} \xi+\eta-u_{1} \eta
\end{array}\right), \\
F_{\left(u_{1}, u_{2}\right)\left(u_{1}, u_{2}\right)}\left(\lambda, u_{1}, u_{2}\right)[\xi, \eta]^{2}=\left(\begin{array}{c}
-2 \lambda \xi \eta \\
-2 \lambda \xi \eta
\end{array}\right) .
\end{gathered}
$$


At $\left(\lambda, u_{1}, u_{2}\right)=\left(k+d \rho_{1}, 0,0\right)$, it is easy to verify that the kernel and the range space are given, respectively, by

$$
\mathcal{N}\left(F_{\left(u_{1}, u_{2}\right)}\left(k+d \rho_{1}, 0,0\right)\right)=\operatorname{span}\{(\psi(x), \omega(x))\}
$$

and

$$
\mathcal{R}\left(F_{\left(u_{1}, u_{2}\right)}\left(k+d \rho_{1}, 0,0\right)\right)=\left\{(f, g) \in Y^{2}: \int_{\Omega} g(x) \omega(x) \mathrm{d} x=0\right\} .
$$

We can also verify that

$$
F_{\lambda\left(u_{1}, u_{2}\right)}\left(k+d \rho_{1}, 0,0\right)[\psi, \omega]=\left(\begin{array}{c}
\omega \\
\omega
\end{array}\right) \notin \mathcal{R}\left(F_{\left(u_{1}, u_{2}\right)}\left(k+d \rho_{1}, 0,0\right)\right) .
$$

Thus, we can apply [9, theorem 1.7] to conclude that the set of positive solutions to $(3.8)$ near $\left(k+d \rho_{1}, 0,0\right)$ is a smooth curve $\Gamma_{1}=\left\{\left(\lambda(s), u_{1}(s), u_{2}(s)\right): s \in(0, \delta)\right\}$ such that $\lambda(0)=k+d \rho_{1}, u_{1}(s)=s \psi+s \phi_{1}(s), u_{2}(s)=s \omega+s \phi_{2}(s)$, where $\phi_{i}$ satisfies the conditions in the theorem. Moreover, $\lambda^{\prime}(0)$ can be calculated (see, for example, [31]) by

$$
\begin{aligned}
\lambda^{\prime}(0) & =-\frac{\left\langle l, F_{\left(u_{1}, u_{2}\right)\left(u_{1}, u_{2}\right)}\left(k+d \rho_{1}, 0,0\right)[\psi, \omega]^{2}\right\rangle}{2\left\langle l, F_{\lambda\left(u_{1}, u_{2}\right)}\left(k+d \rho_{1}, 0,0\right)[\psi, \omega]\right\rangle} \\
& =\frac{2\left(k+d \rho_{1}\right) \int_{\Omega} \psi \omega^{2} \mathrm{~d} x}{2 \int_{\Omega} \omega^{2} \mathrm{~d} x} \\
& =d\left(k+d \rho_{1}\right)^{2} \int_{\Omega} \omega^{3} \mathrm{~d} x,
\end{aligned}
$$

where $l$ is a linear functional on $Y^{2}$ defined as $\langle l,[f, g]\rangle=\int_{\Omega} g(x) \omega(x) \mathrm{d} x$.

(3) (Uniqueness of bifurcation point.) In this part, we prove that $\lambda=k+d \rho_{1}$ is the unique bifurcation value to positive solutions of (3.8) from $(0,0)$. Suppose that there exists a sequence $\left\{\left(\lambda_{n}, u_{1 n}, u_{2 n}\right)\right\}_{n=1}^{+\infty}$ of positive solutions of (3.8) with

$$
\lim _{n \rightarrow+\infty}\left(\lambda_{n}, u_{1 n}, u_{2 n}\right)=(\hat{\lambda}, 0,0) \in \mathbb{R} \times X .
$$

We then find from the second equation of (3.8) with $\lambda=\lambda_{n}$ that, for every $n \geqslant 1$,

$$
-d \Delta\left(\frac{u_{2 n}}{\left\|u_{2 n}\right\|}\right)=\lambda_{n} \frac{u_{2 n}}{\left\|u_{2 n}\right\|}-\lambda_{n} u_{1 n} \frac{u_{2 n}}{\left\|u_{2 n}\right\|}-k \frac{u_{2 n}}{\left\|u_{2 n}\right\|}
$$

or, equivalently,

$d \frac{u_{2 n}}{\left\|u_{2 n}\right\|}=\left(\lambda_{n}-\hat{\lambda}\right)(-\Delta)^{-1}\left(\frac{u_{2 n}}{\left\|u_{2 n}\right\|}\right)+(-\Delta)^{-1}\left(\hat{\lambda} \frac{u_{2 n}}{\left\|u_{2 n}\right\|}-\lambda_{n} u_{1 n} \frac{u_{2 n}}{\left\|u_{2 n}\right\|}-k \frac{u_{2 n}}{\left\|u_{2 n}\right\|}\right)$,

where $\|\cdot\|$ denotes the norm of $Y=L^{r}(\Omega)$. By the compactness of $(-\Delta)^{-1}$, it is easy to see that, along some subsequence, relabelled $n$, we have that

$$
\lim _{n \rightarrow+\infty} \frac{u_{2 n}}{\left\|u_{2 n}\right\|}=\phi>0
$$


for some $\phi \in W^{2, r}(\Omega)$ with $\|\phi\|=1$. Thus, passing to the limit as $n \rightarrow+\infty$ in $(3.10)$, we find that

$$
d \phi=(-\Delta)^{-1}(\hat{\lambda} \phi-k \phi)
$$

or, equivalently,

$$
-d \Delta \phi+k \phi=\hat{\lambda} \phi, \quad x \in \Omega, \quad \phi=0, \quad x \in \partial \Omega .
$$

Therefore, $\hat{\lambda}=k+d \rho_{1}$.

(4) (Global bifurcation.) Recall the Krasnoselskii-Rabinowitz global bifurcation theorem (see, for example, $[29,34]$ ); a connected component $\Sigma_{1}$ of the set of positive solutions in $\mathbb{R}^{+} \times X^{2}$ then contains $\Gamma_{1}$, and $\Sigma_{1}$ satisfies one of the following:

(1) $\Sigma_{1}$ is unbounded;

(2) $\Sigma_{1}$ contains $(\tilde{\lambda}, 0,0)$, where $(\tilde{\lambda}, 0,0)$ is another bifurcation value from which positive solutions bifurcate from $\Gamma_{0}$.

From part (3) of the proof, we know that the second alternative cannot happen. So $\Sigma_{1}$ is unbounded. From part (1) of the proof, if $\lambda \leqslant k+d \rho_{1},(3.8)$ has no positive solution and, from lemma 3.1, any positive solution $\left(u_{1}, u_{2}\right)$ satisfies $u_{1}(x)<1$ and $u_{2}(x)<1 / d$ for any $x \in \Omega$. Hence, the projection of $\Sigma_{1}$ into the $\lambda$-axis must be $\left(k+d \rho_{1}, \infty\right)$. This completes the proof.

We next consider (3.1) with $q>1$. The results are similar to those in theorem 3.2. Hence, we only state the result and point out the difference between the proofs.

THEOREM 3.3. Suppose that $d, k>0$ and $q>1$. Then (3.1) has no positive solution if $\lambda \leqslant d \rho_{1}$, and (3.1) has at least one positive solution if $\lambda>d \rho_{1}$. Moreover, the following hold.

(1) $\lambda=d \rho_{1}$ is a bifurcation value of (3.1), where positive solutions bifurcate from the line of trivial solutions $\Gamma_{0}=\{(\lambda, 0,0): \lambda>0\}$, and $\lambda=d \rho_{1}$ is the unique bifurcation value for which positive solutions bifurcate from $\Gamma_{0}$.

(2) $\operatorname{Near}\left(\lambda, u_{1}, u_{2}\right)=\left(d \rho_{1}, 0,0\right)$, all positive solutions of (3.8) lie on a smooth curve $\Gamma_{1}=\left\{\left(\lambda(s), u_{1}(s, x), u_{2}(s, x)\right): s \in(0, \delta), x \in \Omega\right\}$ for some $\delta>0$, with

$$
\begin{aligned}
& u_{1}(s)=s d \omega+s \phi_{1}(s), \\
& u_{2}(s)=s \omega+s \phi_{2}(s),
\end{aligned}
$$

where $\phi_{1}, \phi_{2}$ are smooth functions from $(0, \delta)$ into a subspace of $X^{2}$ that complements $\operatorname{span}\{(d \omega, \omega)\}$ such that $\phi_{i}(0)=\phi_{i}^{\prime}(0)=0$ for $i=1,2$, and $\lambda(s)$ satisfies

$$
\lambda(0)=d \rho_{1}, \quad \lambda^{\prime}(0)= \begin{cases}\text { does not exist, } & 1<q<2, \\ \left(d^{2} \rho_{1}+k\right) \int_{\Omega} \omega^{3} \mathrm{~d} x, & q=2, \\ d^{2} \rho_{1} \int_{\Omega} \omega^{3} \mathrm{~d} x, & q>2 .\end{cases}
$$


(3) $\Gamma_{1}$ is contained in a connected component $\Sigma_{1}$ of the set of positive solutions of (3.8) in $\mathbb{R}^{+} \times X^{2}$ such that

$$
P_{\lambda} \Sigma_{1}=\left(d \rho_{1}, \infty\right), \quad P_{u_{1}} \Sigma_{1} \subset(0,1), \quad P_{u_{2}} \Sigma_{1} \subset(0,1 / d),
$$

where $P_{\lambda}$ stands for the projection operator into the $\lambda$-component of $\mathbb{R}^{+} \times X^{2}$, and $P_{u_{i}}$ stands for the projection operator into the $u_{i}$-component, $i=1,2$.

Proof. Since the proof is similar to that of theorem 3.2, we omit the details. We only calculate the $\lambda^{\prime}(0)$ here. Define a nonlinear mapping $F: \mathbb{R} \times X^{2} \rightarrow Y^{2}$ by

$$
F\left(\lambda, u_{1}, u_{2}\right)=\left(\begin{array}{c}
\Delta u_{1}+\lambda u_{2}-\lambda u_{1} u_{2} \\
d \Delta u_{2}+\lambda u_{2}-\lambda u_{1} u_{2}-k u_{2}^{q}
\end{array}\right) .
$$

From straightforward calculations, we obtain that

$$
\begin{aligned}
F_{\lambda\left(u_{1}, u_{2}\right)}\left(\lambda, u_{1}, u_{2}\right)[\xi, \eta]= & \left(\begin{array}{l}
-u_{2} \xi+\eta-u_{1} \eta \\
-u_{2} \xi+\eta-u_{1} \eta
\end{array}\right), \\
F_{\left(u_{1}, u_{2}\right)\left(u_{1}, u_{2}\right)}\left(\lambda, u_{1}, u_{2}\right)[\xi, \eta]^{2}= & \left\{\begin{array}{l}
\left(\begin{array}{c}
-2 \lambda \xi \eta \\
-2 \lambda \xi \eta-2 k \eta^{2}
\end{array}\right), \\
\left(\begin{array}{c}
-2 \lambda \xi \eta \\
-2 \lambda \xi \eta-k q(q-1) u_{2}^{q-2} \eta^{2}
\end{array}\right),
\end{array}\right.
\end{aligned}
$$

Moreover, similarly to the proof of theorem $3.2, \lambda^{\prime}(0)$ can be calculated by

$$
\lambda^{\prime}(0)=-\frac{\left\langle l, F_{\left(u_{1}, u_{2}\right)\left(u_{1}, u_{2}\right)}\left(d \rho_{1}, 0,0\right)[d \omega, \omega]^{2}\right\rangle}{2\left\langle l, F_{\lambda\left(u_{1}, u_{2}\right)}\left(d \rho_{1}, 0,0\right)[d \omega, \omega]\right\rangle},
$$

where $l$ is a linear functional on $Y \times Y$ defined as $\langle l,[f, g]\rangle=\int_{\Omega} g(x) \omega(x) \mathrm{d} x$.

If $1<q<2, \lambda(s)$ is not differentiable at $s=0$ and $\lim _{s \rightarrow 0^{+}} \lambda^{\prime}(s)=\infty$, so $\lambda^{\prime}(0)$ does not exist. If $q=2$, we get

$$
\lambda^{\prime}(0)=\frac{2 d^{2} \rho_{1} \int_{\Omega} \omega^{3} \mathrm{~d} x+2 k \int_{\Omega} \omega^{3} \mathrm{~d} x}{2 \int_{\Omega} \omega^{2} \mathrm{~d} x}=\left(d^{2} \rho_{1}+k\right) \int_{\Omega} \omega^{3} \mathrm{~d} x .
$$

If $q>2$, we get

$$
\lambda^{\prime}(0)=\frac{2 d^{2} \rho_{1} \int_{\Omega} \omega^{3} \mathrm{~d} x}{2 \int_{\Omega} \omega^{2} \mathrm{~d} x}=d^{2} \rho_{1} \int_{\Omega} \omega^{3} \mathrm{~d} x
$$

\section{Uniqueness and stability}

In this section, we study the uniqueness and stability of the positive solution of (3.1) in one dimension, i.e.

$$
\left.\begin{array}{cr}
-u_{1}^{\prime \prime}=\lambda u_{2}-\lambda u_{1} u_{2}, & x \in(0, L), \\
-d u_{2}^{\prime \prime}=\lambda u_{2}-\lambda u_{1} u_{2}-k u_{2}^{q}, & x \in(0, L), \\
u_{1}(0)=u_{1}(L)=u_{2}(0)=u_{2}(L)=0
\end{array}\right\}
$$

where $L$ is a positive constant and the double prime denotes $\mathrm{d}^{2} / \mathrm{d} x^{2}=\Delta$. 
We study the uniqueness of the positive solution in two cases: $q=1$ and $q>1$. First, we consider the case of $q=1$, i.e. the system

$$
\left.\begin{array}{cc}
-u_{1}^{\prime \prime}=\lambda u_{2}-\lambda u_{1} u_{2}, & x \in(0, L), \\
-d u_{2}^{\prime \prime}=(\lambda-k) u_{2}-\lambda u_{1} u_{2}, & x \in(0, L), \\
u_{1}(0)=u_{1}(L)=u_{2}(0)=u_{2}(L)=0 .
\end{array}\right\}
$$

By theorem 3.2, (4.1) has a positive solution if and only if $\lambda>k+d \rho_{1}$. In order to get the uniqueness result, we first consider the linear system

$$
\left.\begin{array}{rl}
-u_{1}^{\prime \prime}+\lambda u_{2}^{0} u_{1}=\lambda\left(1-\hat{u}_{1}\right) u_{2}, & x \in(0, L), \\
-d u_{2}^{\prime \prime}+\left(k-\lambda+\lambda u_{1}^{0}\right) u_{2}=-\lambda \hat{u}_{2} u_{1}, & x \in(0, L), \\
u_{1}(0)=u_{1}(L)=u_{2}(0)=u_{2}(L)=0,
\end{array}\right\}
$$

where $\left(u_{1}^{0}, u_{2}^{0}\right)$ is a positive solution of $(4.1)$, and $\hat{u}_{1}(x), \hat{u}_{2}(x) \in C[0, L]$ such that

$$
\hat{u}_{1}(x)<1, \quad \hat{u}_{2}(x)>0, \quad x \in[0, L] .
$$

Then, similarly to [22, theorem 4.1], we have the following result.

Lemma 4.1. Assume that $\left(u_{1}^{0}, u_{2}^{0}\right)$ is a positive solution of (4.1) and $\hat{u}_{1}(x), \hat{u}_{2}(x) \in$ $C[0, L]$ satisfy (4.3). Then $(0,0)$ is the unique solution of (4.2).

Based on the above lemma, we get the following uniqueness result for the case of $q=1$.

THEOREM 4.2. There is a unique positive solution of (4.1) if and only if $\lambda>k+d \rho_{1}$.

Proof. The existence of the positive solution has been proved in theorem 3.2, and hence we only prove the uniqueness. Suppose that $\left(u_{1}, u_{2}\right)$ and $\left(\tilde{u}_{1}, \tilde{u}_{2}\right)$ are two arbitrary positive solutions of (4.1), and let $U_{1}=u_{1}-\tilde{u}_{1}, U_{2}=u_{2}-\tilde{u}_{2}$. Then, $\left(U_{1}, U_{2}\right)$ satisfies

$$
\begin{array}{cc}
-U_{1}^{\prime \prime}+\lambda u_{2} U_{1}=\lambda\left(1-\tilde{u}_{1}\right) U_{2}, & x \in(0, L), \\
-d U_{2}^{\prime \prime}+\left(k-\lambda+\lambda u_{1}\right) U_{2}=-\lambda \tilde{u}_{2} U_{1}, & x \in(0, L), \\
u_{1}(0)=u_{1}(L)=u_{2}(0)=u_{2}(L)=0, &
\end{array}
$$

By lemma 3.1, $\left(\tilde{u}_{1}, \tilde{u}_{2}\right)$ satisfies (4.3). Hence, by using lemma 4.1, $U_{1}=U_{2}=0$ must hold, i.e. $u_{1}=\tilde{u}_{1}$ and $u_{2}=\tilde{u}_{2}$. This completes the proof.

We next consider the uniqueness for the case $q>1$. We first introduce several lemmas, which are used to get the uniqueness. The following lemma is well known.

Lemma 4.3. Assume that $\lambda>d \rho_{1} ;(P)_{0}$ then has a unique positive solution

$$
\left(u_{1}, u_{2}\right)=\left(d \lambda^{-1} \theta_{d / \lambda}, \lambda^{-1} \theta_{d / \lambda}\right),
$$

where $\theta_{a}$ with $a>\rho_{1}$ is the unique positive solution (see [10]) of the problem

$$
\left.\begin{array}{c}
-\phi^{\prime \prime}=\phi(a-\phi), \quad x \in(0, L), \\
\phi(0)=\phi(L)=0 .
\end{array}\right\}
$$


Proof. It is easy to see that $u_{1}=d u_{2}$ from $(P)_{0}$. So, $u_{2}$ satisfies

$$
\left.\begin{array}{c}
-d u_{2}^{\prime \prime}=u_{2}\left(\lambda-\lambda d u_{2}\right), \quad x \in(0, L), \\
u_{2}(0)=u_{2}(L)=0,
\end{array}\right\}
$$

and the unique positive solution of (4.5) is $\lambda^{-1} \theta_{d / \lambda}$.

Lemma 4.4. Assume that $q>1$. Let $\left(u_{1}^{0}, u_{2}^{0}\right)$ be an arbitrary positive solution of $(P)_{k}$; the linearized system of $(P)_{k}$ at $\left(u_{1}^{0}, u_{2}^{0}\right)$ then has only the trivial solution $(0,0)$. Hence, any positive solutions of $(P)_{k}$ are not degenerate.

Proof. The linearized system of $(P)_{k}$ at $\left(u_{1}^{0}, u_{2}^{0}\right)$ is

$$
\left.\begin{array}{rr}
-u^{\prime \prime}+\lambda u_{2}^{0} u=\lambda\left(1-u_{1}^{0}\right) v, & x \in(0, L), \\
-d v^{\prime \prime}+\left(\lambda u_{1}^{0}+k q\left(u_{2}^{0}\right)^{q-1}-\lambda\right) v=-\lambda u_{2}^{0} u, & x \in(0, L), \\
u(0)=u(L)=v(0)=v(L)=0 . &
\end{array}\right\}
$$

If $k=0$, it is obvious that $u=d v$. So, $v$ satisfies

$$
\left.\begin{array}{c}
-d v^{\prime \prime}+\left(\lambda d u_{2}^{0}-\lambda+\lambda u_{1}^{0}\right) v=0, \quad x \in(0, L), \\
v(0)=v(L)=0 .
\end{array}\right\}
$$

Since $\left(u_{1}^{0}, u_{2}^{0}\right)$ is a positive solution of $(P)_{0}$, we have that

$$
\rho_{1}\left(-d \Delta+\lambda d u_{2}^{0}-\lambda+\lambda u_{1}^{0}\right)>\rho_{1}\left(-d \Delta-\lambda+\lambda u_{1}^{0}\right)=0 .
$$

Therefore, $u=v=0$.

Next, we study the case $k>0$. Consider the operators $L_{1}$ and $L_{2}$ defined by

$$
L_{1} \phi=-\phi^{\prime \prime}+\lambda u_{2}^{0} \phi \quad \text { and } \quad L_{2} \phi=-d \phi^{\prime \prime}+\left(\lambda u_{1}^{0}+k q\left(u_{2}^{0}\right)^{q-1}-\lambda\right) \phi, \quad \phi \in \mathcal{X},
$$

where $\mathcal{X}=C_{0}^{2}[0, L]=\left\{u \in C^{2}[0, L]: u(0)=u(L)=0\right\}$. Then $L_{1}$ and $L_{2}$ are invertible. In fact, that $L_{1}$ is invertible follows from $\rho_{1}\left(L_{1}\right)>\rho_{1}>0$. Note that $\left(u_{1}^{0}, u_{2}^{0}\right)$ is a positive solution of $(P)_{k}$; we then have that

$$
\rho_{1}\left(L_{2}\right)>\rho_{1}\left(-d \Delta+\lambda u_{1}^{0}+k\left(u_{2}^{0}\right)^{q-1}-\lambda\right)=0 .
$$

So $L_{2}$ is invertible.

Let

$$
P:=\{\phi \in \mathcal{X}: \phi(x) \geqslant 0, x \in \Omega\}
$$

be the usual cone of positive functions in $\mathcal{X}$, and let $L_{1}^{-1}$ and $L_{2}^{-1}$ be the inverse operators of $L_{1}$ and $L_{2}$, respectively. It is obvious that $L_{1}^{-1}$ and $L_{2}^{-1}$ are compact and strictly order-preserving operators with respect to $P$. Moreover,

$$
L_{i}^{-1}(P \backslash\{0\}) \subset \operatorname{int} P \quad \text { for } i=1,2 .
$$

In terms of $L_{1}$ and $L_{2},(4.6)$ can be written as

$$
L_{1} u=\lambda\left(1-u_{1}^{0}\right) v, \quad L_{2} v=-\lambda u_{2}^{0} u, \quad u, v \in \mathcal{X} .
$$

In this setting we can show that the only solution of (4.10) is $u=v=0$, using a similar proof as in $[4,23]$, which completes the proof. 
A perturbation argument can be used to show that if $(P)_{k}$ with $q>1$ has exactly one positive solution, which is assumed to be non-degenerate, then $(P)_{k+\epsilon}$ also has exactly one positive solution, provided that $\epsilon$ is small enough. For that purpose, we state the following lemma. Since its proof is basically the same as that of $[4$, lemma 5.4], we omit it here.

Lemma 4.5. Assume that $q>1$ and that $(P)_{k}$ has exactly one positive solution $\left(u_{1}^{0}, u_{2}^{0}\right)$, which is not degenerate. There then exists $\epsilon_{0}=\epsilon(\lambda, k)>0$ such that, for every $\epsilon \in\left(-\epsilon_{0}, \epsilon_{0}\right),(P)_{k+\epsilon}$ has exactly one positive solution $\left(u_{1}(\epsilon), u_{2}(\epsilon)\right)$. Moreover, $\left(u_{1}(0), u_{2}(0)\right)=\left(u_{1}^{0}, u_{2}^{0}\right)$ and the mapping $\epsilon \mapsto\left(u_{1}(\epsilon), u_{2}(\epsilon)\right)$, from $\left(-\epsilon_{0}, \epsilon_{0}\right)$ to $P^{2}$, is $C^{1}$, where $P$ is defined in (4.9).

By using lemmas 4.3-4.5, we now state the following uniqueness result for the case of $q>1$, which also completes theorem 1.3. Again the proof is similar to that of $[4$, theorem 5.1], and is thus omitted.

Theorem 4.6. Assume that $q>1$. Problem $(P)_{k}$ then has a unique positive solution if and only if $\lambda>d \rho_{1}$.

To conclude the paper, we discuss the stability of the unique positive solution when $n=1$ by estimating the eigenvalues of the linearized equation. Similar arguments have been used in, for example, $[14,28]$. The local stability of the unique positive solution of $(P)_{k}$ is important for a better understanding of the dynamics of the original reaction-diffusion system (1.2) when $p=1$ and $n=1$, but it is a challenging question in general. Here, we prove the local stability of the unique positive solution of $(P)_{k}$ when $d=1$ and $q=2$, and leave the general case as an open question. The uniqueness result that we have proved implies that 0 cannot be an eigenvalue for the linearized equation when $d=1$, but it does not exclude purely imaginary eigenvalues, which could result in the occurrence of Hopf bifurcations.

For the stability of the uniqueness of the positive solution of $(P)_{k}$ with $d=1$ and $q=2$, we consider the following semilinear elliptic system:

$$
\left.\begin{array}{cc}
-u_{1}^{\prime \prime}=\lambda u_{2}-\lambda u_{1} u_{2}, & x \in(0, L), \\
-u_{2}^{\prime \prime}=\lambda u_{2}-\lambda u_{1} u_{2}-k u_{2}^{2}, & x \in(0, L), \\
u_{1}(0)=u_{1}(L)=u_{2}(0)=u_{2}(L)=0 .
\end{array}\right\}
$$

Let $\left(u_{1}^{0}, u_{2}^{0}\right)$ be the unique positive solution of (4.11). In order to study the stability of $\left(u_{1}^{0}, u_{2}^{0}\right)$, we consider the following linearized eigenvalue problem at $\left(u_{1}^{0}, u_{2}^{0}\right)$ :

$$
\left.\begin{array}{cc}
-\phi^{\prime \prime}=-\lambda u_{2}^{0} \phi+\left(\lambda-\lambda u_{1}^{0}\right) \psi+\mu \phi, & x \in(0, L), \\
-\psi^{\prime \prime}=-\lambda u_{2}^{0} \phi+\left(\lambda-\lambda u_{1}-2 k u_{2}^{0}\right) \psi+\mu \psi, & x \in(0, L), \\
\phi(0)=\phi(L)=\psi(0)=\psi(L)=0 . &
\end{array}\right\}
$$

Here, $\mu$ is an eigenvalue of $(4.12),(\phi, \psi) \in\left[C^{2}(0, L) \cap C[0, L]\right]^{2}$ is a corresponding eigenfunction and $(\phi, \psi) \not \equiv(0,0)$. Note that $\mu$ may be a complex number and $\phi$ and $\psi$ may be complex-valued functions. The solution $\left(u_{1}^{0}, u_{2}^{0}\right)$ is called a stable one if each eigenvalue $\mu$ has positive real part.

Proposition 4.7. Suppose that $\lambda>\rho_{1}$ and $k>0$, and let $\left(u_{1}^{0}, u_{2}^{0}\right)$ be the unique positive solution of (4.11). Then $\left(u_{1}^{0}, u_{2}^{0}\right)$ is stable. 
Proof. Suppose that $\mu$ is an eigenvalue of $(4.12)$, and $(\phi, \psi)$ is a non-trivial eigenfunction. Let $\xi:=\phi-\psi, \eta:=\psi, z_{0}:=u_{1}^{0}-u_{2}^{0}$ and $v_{0}:=u_{2}^{0} ;\left(z_{0}, v_{0}\right)$ is then the unique positive solution of

$$
\left.\begin{array}{rr}
-z^{\prime \prime}=k v^{2}, & x \in(0, L), \\
-v^{\prime \prime}=(\lambda-k v-\lambda d v-\lambda z) v, & x \in(0, L), \\
z(0)=z(L)=v(0)=v(L)=0,
\end{array}\right\}
$$

and $\left(\xi, \eta, z_{0}, v_{0}\right)$ satisfies

$$
\left.\begin{array}{cc}
-\xi^{\prime \prime}=2 k v_{0} \eta+\mu \xi, & x \in(0, L), \\
-\eta^{\prime \prime}=\left(\lambda-2 k v_{0}-2 \lambda v_{0}-\lambda z_{0}\right) \eta-\lambda v_{0} \xi+\mu \eta, & x \in(0, L), \\
\xi(0)=\xi(L)=\eta(0)=\eta(L)=0 . &
\end{array}\right\}
$$

From now on, we denote by $\bar{h}$ the complex conjugate of a complex function $h$. We multiply the second equation of $(4.14)$ by $\bar{\eta}$, and then integrate over $(0, L)$ to obtain that

$$
\begin{aligned}
\int_{0}^{L}\left\{\left|\eta^{\prime}\right|^{2}+\right. & {\left.\left[\lambda\left(v_{0}+z_{0}\right)-\lambda+k v_{0}\right]|\eta|^{2}\right\} \mathrm{d} x } \\
= & -k \int_{0}^{L} v_{0}|\eta|^{2} \mathrm{~d} x-\lambda \int_{0}^{L}\left(v_{0}|\eta|^{2}+v_{0} \xi \bar{\eta}\right) \mathrm{d} x+\mu \int_{0}^{L}|\eta|^{2} \mathrm{~d} x .
\end{aligned}
$$

Since $v_{0}>0$ and satisfies

$$
-v_{0}^{\prime \prime}+\left[\lambda\left(v_{0}+z_{0}\right)-\lambda+k v_{0}\right] v_{0}=0, \quad x \in(0, L), \quad v_{0}(0)=v_{0}(L)=0,
$$

we have that $\rho_{1}\left(-\mathrm{d}^{2} / \mathrm{d} x^{2}+\lambda\left(v_{0}+z_{0}\right)-\lambda+k v_{0}\right)=0$. Moreover, by (3.4), we have that

$$
\begin{aligned}
0 & =\rho_{1}\left(-\mathrm{d}^{2} / \mathrm{d} x^{2}+\lambda\left(v_{0}+z_{0}\right)-\lambda+k v_{0}\right) \\
& =\inf _{\varphi \in H_{0}^{1}(0, L), \varphi \neq 0} \frac{\int_{0}^{L}\left\{\left|\varphi^{\prime}\right|^{2}+\left[\lambda\left(v_{0}+z_{0}\right)-\lambda+k v_{0}\right]|\varphi|^{2}\right\} \mathrm{d} x}{\int_{0}^{L}|\varphi|^{2} \mathrm{~d} x} \\
& \leqslant \frac{\int_{0}^{L}\left\{\left|\eta^{\prime}\right|^{2}+\left[\lambda\left(v_{0}+z_{0}\right)-\lambda+k v_{0}\right]|\eta|^{2}\right\} \mathrm{d} x}{\int_{0}^{L}|\eta|^{2} \mathrm{~d} x}
\end{aligned}
$$

which means that

$$
\int_{0}^{L}\left\{\left|\eta^{\prime}\right|^{2}+\left[\lambda\left(v_{0}+z_{0}\right)-\lambda+k v_{0}\right]|\eta|^{2}\right\} \mathrm{d} x \geqslant 0 .
$$

So, we get from (4.15) that

$$
k \int_{0}^{L} v_{0}|\eta|^{2} \mathrm{~d} x+\lambda \int_{0}^{L}\left(v_{0}|\eta|^{2}+v_{0} \xi \bar{\eta}\right) \mathrm{d} x-\mu \int_{0}^{L}|\eta|^{2} \mathrm{~d} x \leqslant 0
$$


which implies that

$$
k \int_{0}^{L} v_{0}|\eta|^{2} \mathrm{~d} x+\lambda \int_{0}^{L} v_{0}|\eta|^{2} \mathrm{~d} x+\lambda \operatorname{Re}\left(\int_{0}^{L} v_{0} \xi \bar{\eta} \mathrm{d} x\right) \leqslant \operatorname{Re}(\mu) \int_{0}^{L}|\eta|^{2} \mathrm{~d} x .
$$

We estimate the term $\operatorname{Re}\left(\int_{0}^{L} v_{0} \xi \bar{\eta} \mathrm{d} x\right)$. First, using the first equation of (4.14), we have that

$$
\operatorname{Re}(\xi \bar{\eta})=\operatorname{Re}(\eta \bar{\xi})=\frac{1}{2 k}\left(-v_{0}^{-1} \bar{\xi} \xi^{\prime \prime}-v_{0}^{-1} \operatorname{Re}(\mu)|\xi|^{2}\right) .
$$

Hence,

$$
\begin{aligned}
\operatorname{Re}\left(\int_{0}^{L} v_{0} \xi \bar{\eta} \mathrm{d} x\right) & =\frac{1}{2 k} \int_{0}^{L}\left(-\operatorname{Re}(\mu)|\xi|^{2}-\bar{\xi} \xi^{\prime \prime}\right) \mathrm{d} x \\
& =\frac{1}{2 k} \int_{0}^{L}\left(-\operatorname{Re}(\mu)|\xi|^{2}+\left|\xi^{\prime}\right|^{2}\right) \mathrm{d} x .
\end{aligned}
$$

By (4.17) and (4.18), we obtain that

$$
(k+\lambda) \int_{0}^{L} v_{0}|\eta|^{2} \mathrm{~d} x+\frac{\lambda}{2 k} \int_{0}^{L}\left|\xi^{\prime}\right|^{2} \mathrm{~d} x \leqslant \operatorname{Re}(\mu)\left[\int_{0}^{L}\left(|\eta|^{2}+\frac{\lambda}{2 k}|\xi|^{2}\right) \mathrm{d} x\right] .
$$

Since $(\xi, \eta) \not \equiv 0, \operatorname{Re}(\mu)>0$ from (4.19), which proves the stability of $\left(u_{1}^{0}, u_{2}^{0}\right)$.

\section{Acknowledgements}

J.Z. was partly supported by the NSFC (Grant nos 11126141 and 11201380), the China Scholarship Council, the Fundamental Research Funds for the Central Universities (Grant no. XDJK2012B007), the Doctoral Fund (Grant no. SWU111021) and the Educational Fund (Grant no. 2010JY053) of Southwest University. J.S. was partly supported by the NSF (Grant no. DMS-1022648) and the Shanxi 100-Talent Plan Program.

\section{References}

1 G. Arioli. Long term dynamics of a reaction-diffusion system. J. Diff. Eqns 235 (2007), 298-307.

2 J. Blat and K. J. Brown. Bifurcation of steady-state solutions in predator-prey and competition systems. Proc. R. Soc. Edinb. A 97 (1984), 21-34.

3 J. Bricmont, A. Kupiainen and J. Xin. Global large time self-similarity of a thermal-diffusive combustion system with critical nonlinearity. J. Diff. Eqns 130 (1996), 9-35.

4 A. Casal, J. C. Eilbeck and J. López-Gómez. Existence and uniqueness of coexistence states for a predator-prey model with diffusion. Diff. Integ. Eqns 7 (1994), 411-439.

5 K. C. Chang. Methods in nonlinear analysis, Springer Monographs in Mathematics (Springer, 2005).

6 X. F. Chen and Y. W. Qi. Sharp estimates on minimum travelling wave speed of reaction diffusion systems modelling autocatalysis. SIAM J. Math. Analysis 39 (2007), 437-448.

$7 \quad$ X. F. Chen and Y. W. Qi. Propagation of local disturbances in reaction diffusion systems modeling quadratic autocatalysis. SIAM J. Appl. Math. 69 (2008), 273-282.

8 X. F. Chen and Y. W. Qi. Travelling waves of auto-catalytic chemical reaction of general order: an elliptic approach. J. Diff. Eqns 246 (2009), 3038-3057. 
9 M. G. Crandall and P. H. Rabinowitz. Bifurcation from simple eigenvalues. J. Funct. Analysis 8 (1971), 321-340.

10 E. N. Dancer. On positive solutions of some pairs of differential equations. Trans. Am. Math. Soc. 284 (1984), 729-743.

11 E. N. Dancer, J. López-Gómez and R. Ortega. On the spectrum of some linear non-cooperative elliptic systems with radial symmetry. Diff. Integ. Eqns 8 (1995), 515-523.

12 K. Deimling. Nonlinear functional analysis (Springer, 1985).

13 Y. H. Du. Bifurcation and related topics in elliptic problems. In Handbook of differential equations: stationary partial differential equations, vol. II, pp. 127-209 (Elsevier, 2005).

14 Y. Du and J. Shi. A diffusive predator-prey model with a protection zone. J. Diff. Eqns 229 (2006), 63-91.

15 I. R. Epstein, H. Rubinstein and J. A. Pojman. An introduction to nonlinear chemical dynamics: oscillations, waves, patterns, and chaos, Topics in Physical Chemistry (Oxford University Press, 1998).

16 P. Gray and S. Scott. Oscillations and instabilities: nonlinear chemical kinetics, International Series of Monographs on Chemistry (Oxford: Clarendon, 1990).

17 E. Jakab, D. Horváth, J. H. Merkin, S. K. Scott, P. L. Simon and A. Tóth. Isothermal flame balls. Phys. Rev. E 66 (2002), 016207.

18 E. Jakab, D. Horváth, J. H. Merkin, S. K. Scott, P. L. Simon and A. Tóth. Isothermal flame balls: effect of autocatalyst decay. Phys. Rev. E 68 (2003), 036210

19 J. F. Jiang and J. P. Shi. Dynamics of a reaction-diffusion system of autocatalytic chemical reaction. Discrete Contin. Dynam. Syst. 21 (2008), 245-258.

20 Y. Li and Y. W. Qi. The global dynamics of isothermal chemical systems with critical nonlinearity. Nonlinearity 16 (2003), 1057-1074.

$21 \mathrm{Y}$. Li and Y. Wu. Stability of traveling front solutions with algebraic spatial decay for some autocatalytic chemical reaction systems. SIAM J. Math. Analysis 44 (2012), 1474-1521.

22 J. López-Gómez. The steady states of a non-cooperative model of nuclear reactors. J. Diff. Eqns 246 (2009), 358-372.

23 J. López-Gómez and R. Pardo. Existence and uniqueness of coexistence states for the predator-prey model with diffusion: the scalar case. Diff. Integ. Eqns 6 (1993), 1025-1031.

24 A. J. Lotka. Elements of mathematical biology (New York, NY: Dover, 1958).

25 G. Nicolis and I. Prigogine. Self-organization in nonequilibrium systems: from dissipative structures to order through fluctuations (Wiley, 1977).

26 T. C. Ouyang and J. P. Shi. Exact multiplicity of positive solutions for a class of semilinear problems. J. Diff. Eqns 146 (1998), 121-156.

27 T. C. Ouyang and J. P. Shi. Exact multiplicity of positive solutions for a class of semilinear problem. II. J. Diff. Eqns 158 (1999), 94-151.

28 R. Peng, D. Wei and G. Y. Yang. Asymptotic behaviour, uniqueness and stability of coexistence states of a non-cooperative reaction-diffusion model of nuclear reactors. Proc. $R$. Soc. Edinb. A 140 (2010), 189-201.

29 P. H. Rabinowitz. Some global results for nonlinear eigenvalue problems. J. Funct. Analysis 7 (1971), 487-513.

30 J. Schnakenberg. Simple chemical reaction systems with limit cycle behaviour. J. Theor. Biol. 81 (1979), 389-400.

31 J. P. Shi. Persistence and bifurcation of degenerate solutions. J. Funct. Analysis 169 (1999), 494-531.

32 J. P. Shi and R. Shivaji. Persistence in reaction diffusion models with weak Allee effect. J. Math. Biol. 52 (2006), 807-829.

33 J. P. Shi and X. F. Wang. Hair-triggered instability of radial steady states, spread and extinction in semilinear heat equations. J. Diff. Eqns 231 (2006), 235-251.

34 J. P. Shi and X. F. Wang. On global bifurcation for quasilinear elliptic systems on bounded domains. J. Diff. Eqns 246 (2009), 2788-2812.

35 V. Volterra. Fluctuations in the abundance of a species considered mathematically. Nature 119 (1927), 12-13.

36 Q. Y. Ye and Z. Y. Li. Introduction to reaction-diffusion equations (Beijing: Science Press, 1990).

37 Y. Zhao, Y. Wang and J. P. Shi. Steady states and dynamics of an autocatalytic chemical reaction model with decay. J. Diff. Eqns 253 (2012), 533-552.

(Issued 4 April 2014) 